\title{
Enabling component technologies for space division multiplexing
}

\author{
Y. Jung, ${ }^{*}$ S. U. Alam and D. J. Richardson \\ Optoelectronics Research Centre, University of Southampton, Southampton, SO17 1BJ, UK \\ *ymj@orc.soton.ac.uk
}

\begin{abstract}
We present an overview of recent progress on SDM components. In particular, we will discuss in detail various recently developed SDM fiber isolators, pump couplers and mode field diameter adaptors.

OCIS codes: (060.2340) Fiber optics components; (230.1150) All-optical devices.
\end{abstract}

\section{Introduction}

In order to fulfil the vision of fully integrated Space Division Multiplexed (SDM) transmission [1], the development of a wide range of passive SDM components offering the benefits of relatively low material costs, simple fabrication procedures and highly integrated solutions is very important. Unlike widely used single-mode fiber systems, many essential components needed to build SDM transmission systems are still not commercially available and basic prototype systems used in many experiments to date have been implemented only with the aid of numerous freespace optical components positioned on relatively large optical benches. These are not only bulky but also expensive and introduce high optical losses. From this perspective, a fiber-optic approach will inevitably be the preferred route forward and the fabrication of fully fiberized components is a pre-requisite for the realization of practical SDM systems. In this paper, we will focus on several interesting SDM components that are essential to realize fully integrated SDM transmission; in particular, fiber optic collimator assemblies, pump couplers, mode field diameter adaptors and mode dependent loss equalizers.

\section{SDM optical components}

\section{1) SDM fiber islolators}

An optical fiber collimator assembly represents an important platform for the realization of many commonly used fiber optic components and devices including: optical isolators, circulators, gain flattening filters, WDM couplers, and so on. Here, SDM fibers (e.g. few mode fibers or multicore fibers) can be incorporated in to a micro-optic collimator assembly and compact SDM components can readily be realized [2]. Due to the simple fabrication process, good beam transfer quality and low cost, these devices represent an extremely attractive means of reducing the cost of building and operating SDM systems. As shown in Fig. 1(top), compact fiber optic collimators (typically GRIN lens or C-lens based) are used to transform the emergent light from an input SDM fiber into a collimated free

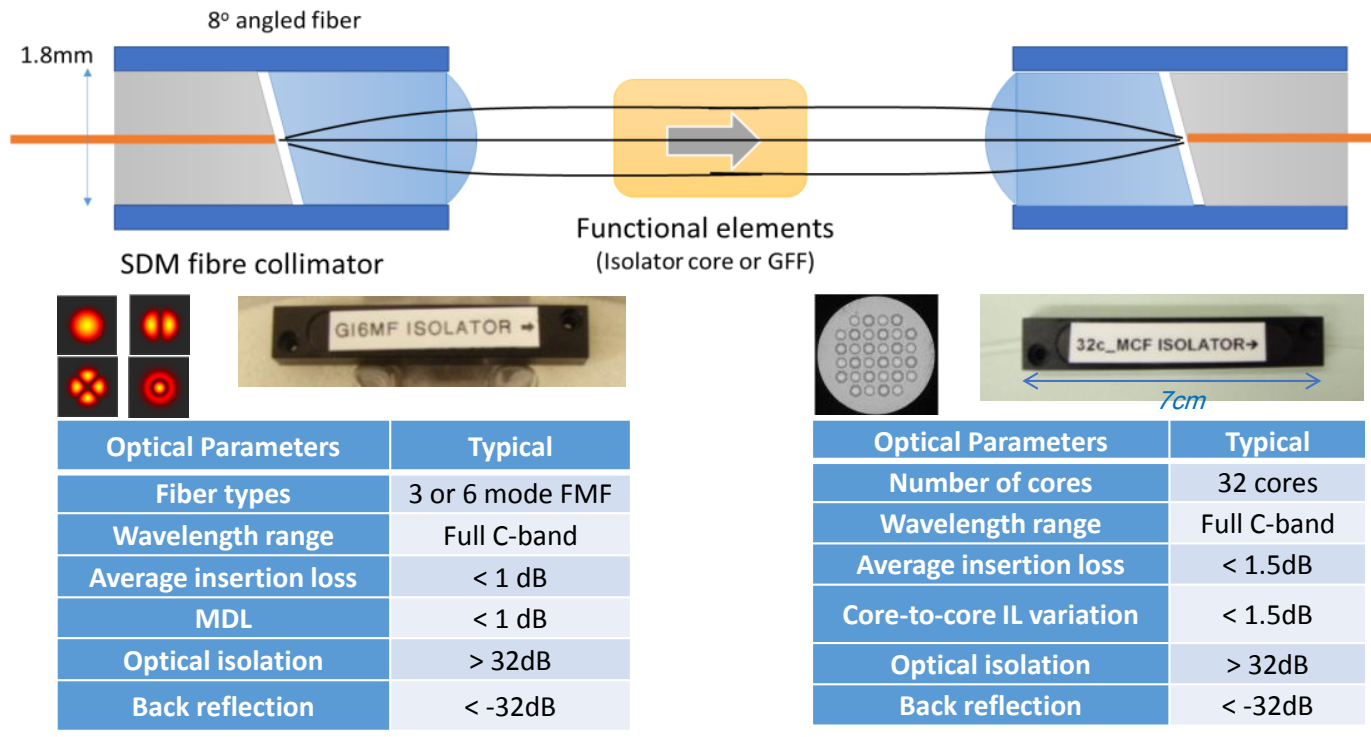

Fig. 1. Schematic of a SDM fiber isolator using compact fiber optic collimators (top) and associated tables summarizing the key performance of both few mode and multicore fiber isolator (bottom). 
space beam that can then be refocused into another SDM fiber using a second identical assembly but in reverse. Optical elements (e.g. an isolator core, bandpass filter, gain flattening filter and so on) can then be inserted into the free space region to provide in-line functionality. To illustrate the capability we note that excellent optical performance in terms of average insertion loss $(<1 \mathrm{~dB})$ and mode dependent loss $(<1 \mathrm{~dB})$ has readily been demonstrated for both 3-mode and 6-mode FMF isolators. The maximum peak isolation was more than 40dB and optical isolation over the full C-band was more than $30 \mathrm{~dB}$. This approach can be applicable to other types of SDM fibers, for example a high-core-count 32-core multicore fiber isolator has also been recently demonstrated [2]. Unlike FMF collimators, MCF collimators intrinsically require rotational alignment and a multi-axis precision micro-stage is generally used to align the collimators prior to gluing. The optical performance is most encouraging with an average insertion loss of $\sim 1.5 \mathrm{~dB}$, core-to-core variation of $\sim 1.5 \mathrm{~dB}$ and an inter-core crosstalk of less than $40 \mathrm{~dB}$. This indicates that the fiber optic collimator assembly concept can be extended to SDM fibers, providing the opportunity for an array of new and practical packaged components with performance, in terms of functionality and insertion loss, comparable to the equivalent existing single mode fiber devices, whilst at the same time ensuring low levels of inter-channel cross-talk.

\section{2) Optical fiber pump coupler}

Pump couplers (or WDM couplers) have been widely used to efficiently couple pump light ( $\lambda=980$ or $1480 \mathrm{~nm}$ ) into the active fiber along with the signal. Fused fiber coupler and dichroic mirror coupler technologies are the most commonly used methods to fabricate SMF pump couplers. However, the fused fiber coupler approach is not considered as suitable for SDM applications because each spatial mode (core) experiences different coupling efficiency, resulting in mode (core) dependent loss. Therefore, a dichroic mirror based fiber optic collimator assembly is the preferred approach for SDM applications, and low mode (core) dependent loss can be achieved with this technique. The schematic of a typical dichroic mirror coupler is shown in Fig. 2(a). A dichroic mirror is placed in the optical path between the two collimators (i.e. signal input and output collimators) without disturbing the signals and combines the pump and signal light onto the same optical path. Note that this approach is very efficient for core-pumped amplifiers but is not suitable for cladding-pumped amplifiers as it requires a high power handling capability (up to a few tens of Watts). Recently, a fully-fiberized side pump coupler has been employed to couple multimode pump radiation into an active SDM fiber as shown in Fig. 2(b). A multimode pump delivery fiber (core/clad diameter $=105 / 125 \mu \mathrm{m}$, numerical aperture $=0.22$ which is compatible with that used on most multimode $976 \mathrm{~nm}$ pump diodes) was tapered down to $15 \mu \mathrm{m}$ with a uniform taper length of $20 \mathrm{~mm}$ and wound with a slight tension around a short stripped section of the active SDM fiber. Although a pump coupling efficiency of $\sim 70 \%$ can easily be achieved, it is possible to increase the coupling efficiency to values in excess of $90 \%$ using slightly more sophisticated approaches. A UV-curable low-index polymer can be applied to the tapered section and then cured to ensure robust, stable optical contact within the pump combiner. There is no observable change in pump coupling efficiency as a result of this packaging. Also, note that multiple side pump couplers can be employed without access to the core of the signal fiber and this can be used to substantially improve the uniformity of population inversion along the fiber length.

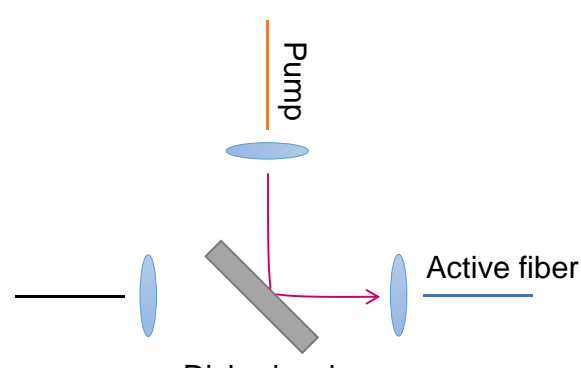

Dichroic mirror

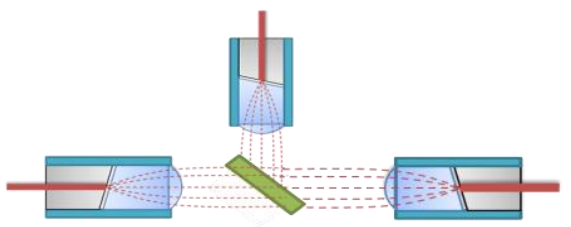

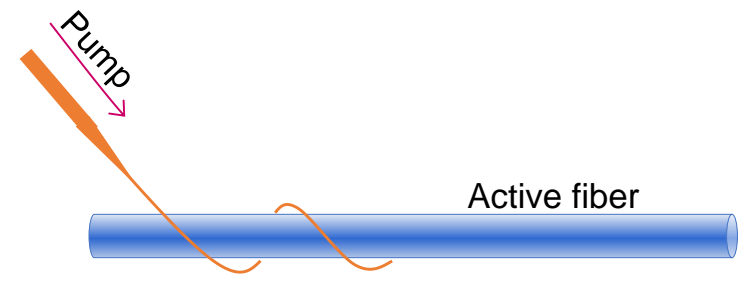

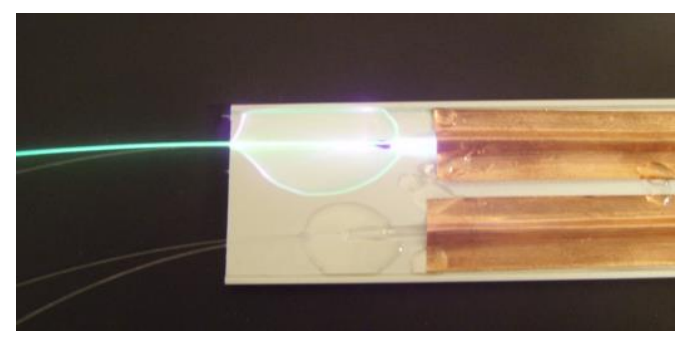

Fig. 2. Schematic of pump couplers for (a) core-pumped and (b) cladding-pumped SDM amplifiers. 


\section{3) Mode field diameter adaptor}

Another important issue for successful SDM transmission is the optical interconnection between dissimilar SDM fibers. For example, even in the same category of few mode fibers, multiple fiber designs exist with different mode field diameters (MFDs). Generally, the MFD mismatch results in a mode dependent splice loss for each spatial mode and this splice loss variation directly results in an optical signal to noise ratio variation between spatial modes, ultimately limiting the overall SDM transmission performance. To resolve this issue, a very simple and compact allfiber structure for low loss optical interconnection of dissimilar FMFs is highly desirable. Recently, a graded index fiber (GIF) based MFD adaptor has been introduced [3] and the mode dependent splice loss was significantly reduced. Use of a GIF based MFD adaptor was previously introduced to accommodate the MFD mismatch between two dissimilar single mode fibers and to reduce the splice loss, and the same technology can be employed for low loss optical interconnection between dissimilar FMFs. Figure 3(a) shows the basic geometry of an all-fiber mode adaptor using a graded index fiber and a coreless fiber (CSF), where the GIF is used as an effective lens element and a segment of CSF is used as a spacer. By choosing an appropriate length of both fibers, a compact all-fiber MFD adaptor can easily be realized using only a simple cleaving and fusion splicing procedure. To prove the technique two FMFs having significantly different MFDs were taken and the mode dependent splice loss was investigated. FMF1 had a core diameter of $20 \mu \mathrm{m}$, an NA of 0.12 and FMF2 has a core diameter of $10 \mu \mathrm{m}$ and an NA of 0.22 . As shown in Fig. 3(b), the direct splice loss is expected to be $\sim 2.2 \mathrm{~dB}$ for the $\mathrm{LP}_{01}$ and $4.0 \mathrm{~dB}$ for $\mathrm{LP}_{11}$ modes respectively. However, using the proposed all-fiber mode adaptor these values can be reduced to less than $0.15 \mathrm{~dB}$ for both spatial modes. Moreover, this technique can also be used for dissimilar multicore fiber interconnection, where a substantial core-pitch difference between dissimilar MCFs can be effectively compensated by employing this simple all-fiber structure [3]. These examples clearly show that such all-fiber mode adaptors can provide great control and efficiency in optical coupling for a plurality of different SDM fibers and applications.

(a)

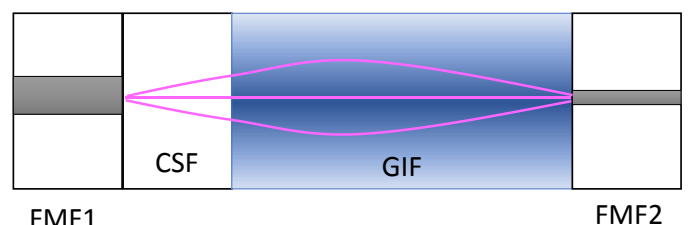

(d1=20um, NA=0.12)

(d2=10um, NA=0.22)

(b)

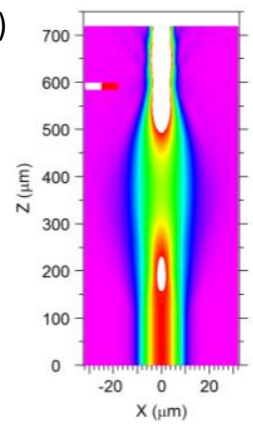

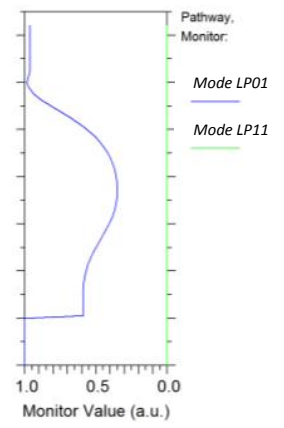

$(\mathrm{LP} 01)$
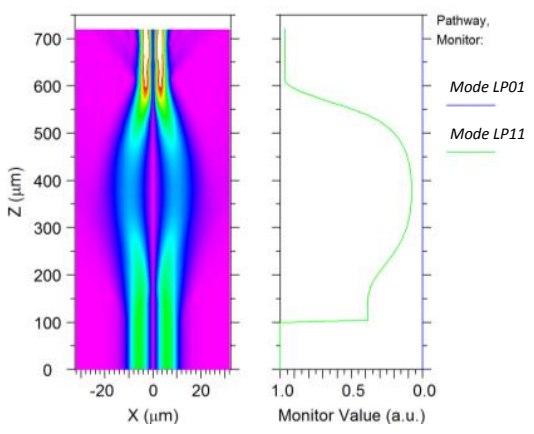

$\left(\mathrm{LP}_{11}\right)$

Fig. 3. (a) All-fiber mode field diameter adaptor for low mode dependent splice loss between dissimilar FMFs and (b) beam propagation method (BPM) simulation of the proposed mode adaptor.

\section{Conclusion}

Significant research advances have been made recently in the development of SDM components with reasonable insertion losses ( 1 or $2 \mathrm{~dB}$ ). Fiberized side-pumping schemes have been demonstrated, along with integrated passive SDM components such as isolators and filters, which promise significant cost savings relative to the use of an equivalent numbers of conventional single mode fiber components.

This work was supported by the EU-Japan coordinated R\&D project on "Scalable And Flexible optical Architecture for Reconfigurable Infrastructure (SAFARI)" by the Ministry of Internal Affairs and Communications (MIC) of Japan and EC Horizon 2020 and by the EPSRC funded "Hollow Core Fiber Photonics" Programme Grant (EP/P030181/1).

\section{References}

[1] D. J. Richardson, et al., "Space-division multiplexing in optical fibres," Nat. Photonics 7(5), 354-362 (2013).

[2] Y. Jung et al., "Compact 32-core multicore fibre isolator for high-density spatial division multiplexed transmission," ECOC'16, W2.B4.

[3] Y. Jung et al., “All-fiber optical interconnection for dissimilar multicore fibers with low insertion loss," OFC'17, W3H.2. 